\title{
GUAYAQUIL: INTEGRACIÓN TERRITORIAL HISTÓRICA Y TENDENCIAS CONTEMPORÁNEAS
}

\author{
GUAYAQUIL: HISTORICAL TERRITORIAL INTEGRATION \\ AND CONTEMPORARY TRENDS
}

\section{RUBÉN AROCA JÁCOME}

Universidad Católica de Santiago de Guayaquil. carlos.aroca@cu.ucsg.edu.ec

RESUMEN

La integración territorial de la zona que actualmente corresponde al área urbana de Guayaquil, así como a sus áreas de influencia, no inicia con la modernidad. Este proceso se produce en distintas etapas, desde lo precolonial hasta lo contemporáneo, y va construyendo vínculos duraderos que traspasan la institucionalidad estatal. A partir de la relación inicial entre los vestigios arqueológicos pre colombinos y las demarcaciones políticas del presente, sostenemos que los patrones de asentamiento moderno se relacionan con las formas nativas de gestión del territorio, proceso que no encontraría complicaciones estructurales sino hasta bien entrado el siglo XIX, con el fuerte impulso demográfico de Guayaquil y el inicio de un agresivo proceso de urbanización que produciría las ciudades que pueden observarse en el presente.

PALABRAS CLAVE: Integración territorial, urbanización, asentamientos humanos.

\begin{abstract}
The territorial integration of the space that currently corresponds to the urban area of Guayaquil, as well as to its areas of influence, does not begin with modernity. This process takes place in different stages, from natives communities (pre-hispanish) to the contemporary cities, making lasting relations that go beyond the state institutions. Starting from the initial relationship between the pre-Columbian archaeological vestiges and the political boundaries of the present, we argue that the patterns of modern settlement are related to the native forms of territorial management, a process that would not find structural complications until the 19th century, with the strong demographic impulse of Guayaquil, the very beginning of an aggressive urbanization that would produce the cities that we can observed in nowdays.
\end{abstract}

KEYWORDS: Territorial integration, Urnanization, Human Settlement . 
PERSPECTIVA GENERAL

La historia territorial relacionada con el área urbana de Guayaquil es de larga data. Este proceso de integración, es responsable de la estructuración de vínculos duraderos entre dicho espacio y su entorno, contempla tanto el conjunto de relaciones sociales históricamente desarrolladas, una modalidad de producción agrícola que aprovechaba las redes hidrográficas, así como ciertas modalidades de ocupación del suelo que actualmente forman los cantones en el área de influencia de la ciudad.

En efecto, si bien es históricamente cierto que la localización actual de Guayaquil (desde un poco antes de mediados del siglo XVI) es producto de una decisión administrativamilitar-comunicacional en el marco de una gestión sobre las colonias y que, por tanto, ese espacio no suponía una centralidad cultural nativa pre-colonial; no es menos cierto que una buena parte de ese mismo espacio, así como sus actuales zonas de jerarquía superior constituyeron un sistema administrativo pre-existente a la colonia (Cieza de León, 2005). En el marco de lo contemporáneo, algunos de los desarrollos presentes pueden interpretarse como ciertos niveles de continuidad en la gestión del espacio, aunque procesos emergentes, tales como el Puerto de Aguas Profundas de Posorja y el Aeropuerto del Daular, dejen entrever nuevas tendencias en las principales variables urbanas.

\section{ELEMENTOS METODOLÓGICOS}

Partiendo de entender la construcción de relaciones sociales como un proceso de simbolización que se marca en el territorio, se adoptó de modo operativo, para el presente trabajo, los siguientes criterios:

a. Las áreas con significativa concentración de bienes culturales son, a su vez, áreas de constante interacción entre la población y el espacio;

b. Entorno que se percibe como altamente integrado, y las poblaciones o asentamientos humanos que se auto-perciben en una interrelación con el territorio;

c. Espacios demarcados para proyectos de desarrollo contemporáneos.

El estudio observó el estado situacional descrito por el Sistema de Información (ABACO) del Instituto Nacional de Patrimonio Cultural (INPC, 2017), desarrollando una estadística descriptiva y una cartografía (geo referenciación) de los bienes culturales, e iniciando un ejercicio interpretativo de la relación entre estos objetos y el territorio.

En el análisis de los datos se pudieron establecer los ríos y esteros que están asociados a los sitios arqueológicos. Lo anterior permitió relacionar, por ejemplo, población, recursos hidrológicos y bienes culturales. Algunas de las variables utilizadas por el estudio se muestran a continuación en la Tabla 1.

TABLA 1. VARIABLES, DEFINICIONES Y CRITERIOS OPERATIVOS UTILIZADOS EN EL ESTUDIO

\begin{tabular}{|c|c|c|}
\hline VARIABLE & DEFINICIÓN & CRITERIO OPERATIVO \\
\hline Usos de suelo & $\begin{array}{l}\text { Toda forma de } \\
\text { utilización humana del } \\
\text { territorio, en especial } \\
\text { aquellas de tipo habita- } \\
\text { cional, económico, } \\
\text { comunicaciones, etc. }\end{array}$ & $\begin{array}{l}\text { Tipos de Uso } \\
\text { Área utilizada }\end{array}$ \\
\hline Hidrología & $\begin{array}{l}\text { Recursos hidrológicos } \\
\text { de la zona de estudio }\end{array}$ & $\begin{array}{l}\text { Ríos simples } \\
\text { Ríos dobles } \\
\text { Esteros } \\
\text { Áreas estimadas }\end{array}$ \\
\hline $\begin{array}{l}\text { Crecimiento } \\
\text { poblacional }\end{array}$ & $\begin{array}{l}\text { Dinámica demográfica } \\
\text { del área de estudio }\end{array}$ & $\begin{array}{l}\text { Número de habitantes } \\
\text { Número de hombres } \\
\text { Número de mujeres } \\
\text { Grupos de edad }\end{array}$ \\
\hline $\begin{array}{l}\text { Desarrollo } \\
\text { socioeconó- } \\
\text { mico }\end{array}$ & $\begin{array}{l}\text { Actividades orientadas } \\
\text { económicamente }\end{array}$ & $\begin{array}{l}\text { Agricultura } \\
\text { Ganadería } \\
\text { Industria } \\
\text { Comercio } \\
\text { Otras actividades }\end{array}$ \\
\hline $\begin{array}{l}\text { Patrones de } \\
\text { ocupación del } \\
\text { suelo }\end{array}$ & $\begin{array}{l}\text { Asentamientos } \\
\text { humanos permanentes }\end{array}$ & $\begin{array}{l}\text { Ribereños } \\
\text { Ribereños de ambas } \\
\text { márgenes } \\
\text { Ribereños de una sola } \\
\text { margen } \\
\text { En función de zonas de } \\
\text { cultivos } \\
\text { En función de vías o } \\
\text { carreteras } \\
\text { En función de centros } \\
\text { administrativos } \\
\text { Otros tipos de patrones }\end{array}$ \\
\hline $\begin{array}{l}\text { Concentración } \\
\text { de bienes } \\
\text { culturales }\end{array}$ & $\begin{array}{l}\text { Inventario de bienes } \\
\text { ABACO }\end{array}$ & $\begin{array}{l}\text { Proporción de bienes } \\
\text { concentrados por zonas. } \\
\text { Georreferenciación. }\end{array}$ \\
\hline
\end{tabular}

Para el abordaje de ciertos problemas contemporáneos, como los asentamientos de la nueva periferia pobre del área urbana, se analizaron informes oficiales y leyes relacionadas. No obstante, para fines de análisis general se ha preferido no optar por las definiciones institucionales de tipos de áreas según el Gobierno Autónomo Descentralizado (GAD) Municipal de Guayaquil sino que, en su lugar, se han determinado zonas según datos empíricos de interés para el presente estudio. 
RESULTADOS Y DISCUSIÓN

Como se ha anotado anteriormente, ciertas marcas en el territorio (tales como restos arqueológicos) dan cuenta de las tendencias de ocupación del suelo en la época pre-hispánica (INPC, 2017).

La existencia de un complejo de yacimientos y tolas (formaciones de construcción nativa, no naturales, con fines habitacionales, logísticos o funerarios) en la cuenca baja del Guayas cercana a los ríos, deja comprender un patrón tradicional de ocupación territorial para las poblaciones de la región; así como una permanente gestión de un fenómeno recurrente: las inundaciones. En este caso, las poblaciones manejaban tanto las crecidas de los ríos como los fuertes inviernos edificando sobre la cota de inundación (nivel máximo hasta donde puede alcanzar el nivel del agua). No es deleznable o poco plausible,

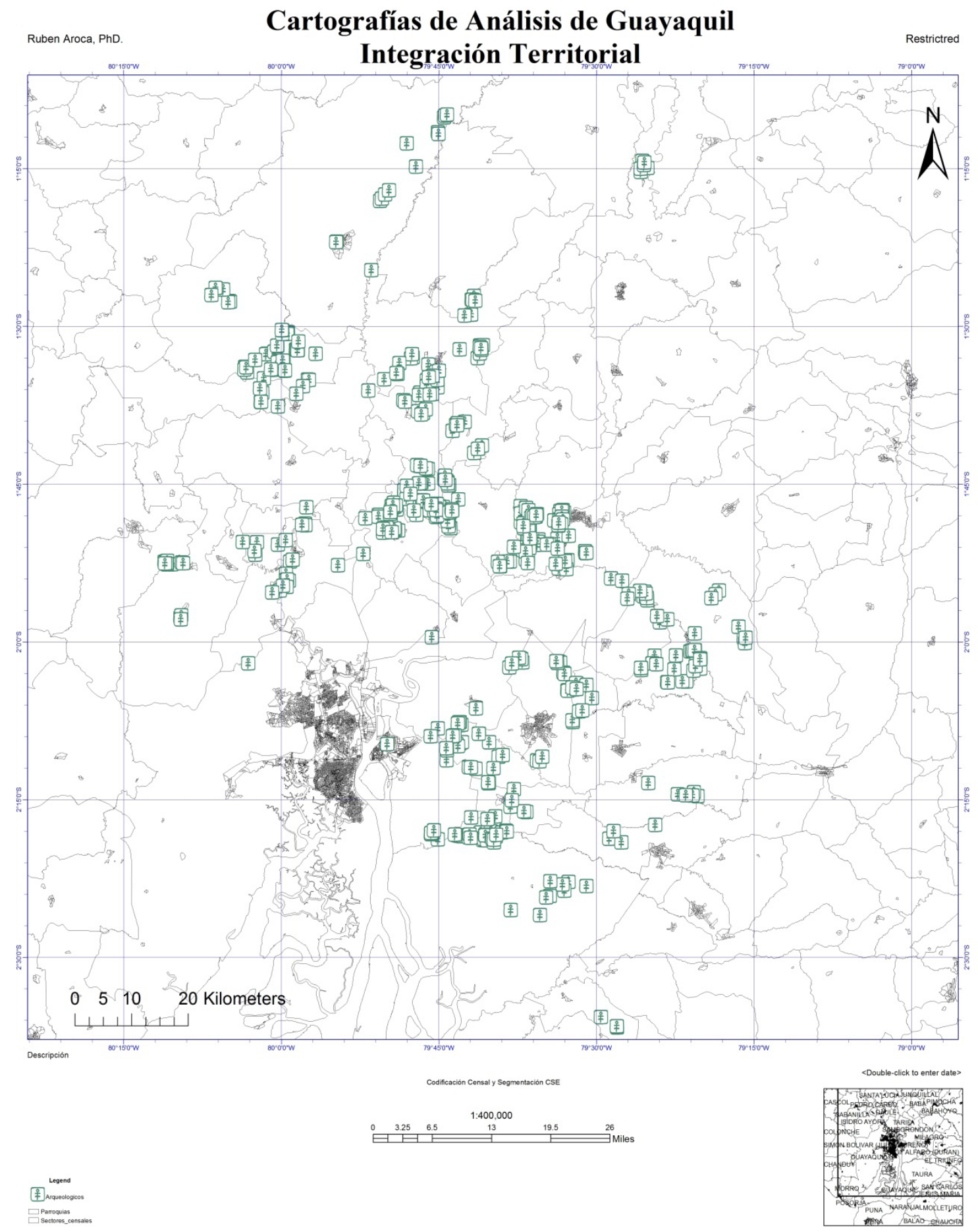

Figura 1. Vestigios arqueológicos en el entorno del área urbana de Guayaquil Fuente: Elaborada a partir de la data del sistema ABACO- INPC. 
por tanto, la hipótesis que se propone de que la integración regional supone sistemas administrativos, agrícolas y de gestión de inundaciones comunes que, de uno u otro modo, se reproducen en las etapas indígena, colonial, moderna y contemporánea.

Las relaciones de espacio de las zonas de la Cuenca Baja se desarrollaron siglos antes de la colonia, en especial bajo Cultura Milagro - Quevedo (Chono) y articuló, ya en el período hispánico, a un nuevo centro administrativo: Guayaquil (Figura 1). Para el siglo XVII, una buena parte del área que presumiblemente correspondió al Reino Chono se encontraba administrativamente integrada como la Provincia de Guayaquil, proceso que sostuvo los vínculos precoloniales y los lanzó a una modernidad particular, la latinoamericana, en forma de relaciones y comunicación inscritas en el suelo, ya en la época de la República y con una nueva estructura político - territorial (Espinoza Soriano, 1999).

Se puede comprender, sobre la base de lo anterior, que todas estas elaboraciones históricas sostuvieron, al menos de modo parcial, tanto los patrones de asentamiento tradicionales así como las formas nativas de manejo de los duros inviernos y las crecidas de ríos, proceso que no encontraría complicaciones estructurales sino hasta bien entrado el siglo XIX, con el fuerte impulso demográfico de Guayaquil y el inicio de un agresivo proceso de urbanización que produciría las ciudades que pueden observarse en el presente.

De este modo, en lo particular y relativo al manejo de inundaciones que las poblaciones del área desarrollaron hasta el siglo XIX, se observa centralmente que aquello no residió en la prác tica de un patrón de asentamiento poblacional que supusiera significativas distancias de los ríos. En efecto, los núcleos urbanos siempre se localizaron muy próximos a las riberas, siendo más bien una ventaja para su expansión, que una dificultad que produjera o desalentara las prácticas recurrentes.

Daría la impresión de que la clave se encuentra más bien en el uso de ciertas formas constructivas que suponían un conocimiento adecuado de las cotas de inundación, procesos que, al parecer, fueron cediendo con la llegada de las ciudades modernas y la agricultura industrial.

\section{PROCESO DEMOGRÁFICO Y VULNERABILIDAD DEL ENTORNO}

Los cantones del área de interés para el presente artículo sostienen tendencias demográficas interrelacionadas, al menos, desde fines del siglo XIX. Esto significa que cualquier política expresada como conjunto de proyectos implementados en la micro-región afectaría, en determinado modo, a todas las unidades territoriales del entorno de influencia directa.

Hamerly (2006), analizando datos de los censos aplicados en Quito y Guayaquil desde 1899 hasta inicios del siglo XX sostiene que, en ambos casos, la dinámica demográfica de las principales ciudades del Ecuador no se puede explicar por el movimiento o mejora en las variables de natalidad y mortalidad.

En efecto, para finales de la década de 1880 la expectativa de vida en Guayaquil era inferior a los 40 años de edad, y este indicador sugiere que la tasa de mortalidad en general, e infantil en particular, era elevada por lo que no era posible, por estos factores, la producción de la moderna urbe tal y cual como se había podido observar tanto en ciudades europeas como americanas. A pesar de aquello, para esa época, Guayaquil se convirtió en la ciudad más poblada del país con 66.531 habitantes; mientras que Quito no superaba los 56.000 ya en 1906 (Hamerly, 2006).

Lo que explica este proceso son los vigorosos flujos migratorios desde los cantones interiores hasta la zona urbana, producidos en dicho siglo y relacionados con la producción agrícola en dichos territorios. Desde esa época, la gestión moderna de la política y la economía crean la ciudad y sus zonas de influencia (área conurbana). Impulso que, a pesar de que se ha ralentizado, no se ha detenido hasta el día de hoy.

La jerarquía territorial se expresa, por tanto, en dos significativos movimientos: a) densificación del suelo urbano; y b) consolidación del suelo urbano.

En la zona de estudio, las dinámicas del espacio se expresan en su densidad demográfica y esto es válido tanto para la zona urbana como para sus áreas de influencia. No obstante, una mayor densidad demográfica no implica por sí sola una mayor jerarquía territorial. Esta hipótesis debe ser matizada por un movimiento contrario; esto es, que mientras menor jerarquía contempla el suelo urbano es más vulnerable a distintos tipos de procesos naturales y antrópicos, entre ellos las inundaciones periódicas.

Es necesario destacar que los patrones de ocupación del suelo hasta fines del 1960, rara vez estuvieron guiados por una política de organización espacial definida desde los gobiernos municipales o centrales. Para Guayaquil y su área conurbana, el ordenamiento territorial es un fenómeno de política relativamente reciente 
y su aplicación nunca resultó una tarea sencilla; siendo frecuentemente interrumpida por el clientelismo electoral.

Como es conocido, una de las características principales que se encuentra en la llanura aluvial de la cuenca del Río Guayas, es la reducción constante de la capacidad de los cauces. Esta última, se produce como consecuencia de la pérdida de turbulencia del flujo debido a la considerable disminución de la pendiente de los cauces y por efecto de la influencia de mareas que aceleran aún más el proceso de deposición del sedimento. A la fecha, no se cuenta con datos que permitan cuantificar la tasa de deposición en el estuario, pero lo que sí resulta evidente es la aparición de depósitos frente a la ciudad de Guayaquil.

Este problema actualmente se encuentra agravado por el incremento anual de arrastre de sedimento como consecuencia de la presión sobre el suelo urbano adyacente a los ríos Daule, Babahoyo y Guayas, así como la despiadada tala de árboles en las fuentes de sedimento; ocasionando en las zonas de poca pendiente una sobrecarga imposible de ser transportada por la corriente de agua, produciendo la deposición de material y complejizando de esta manera el problema de las inundaciones.

TENDENCIAS DE EXPANSIÓN URBANA

Como se ha indicado anteriormente, Guayaquil es el centro urbano de la región (provincias de la costa) y, en mayor o menor medida los cantones, parroquias o poblaciones asentadas en estos territorios dependen de lo que suceda en la ciudad. Por ello, en el marco del presente trabajo, se pretenden identificar tendencias de desarrollo, tanto en cuanto al uso del suelo como en lo referente a la densificación demográfica.

Desde el punto de vista de nuestras preocupaciones, proponemos identificar las siguientes zonas de desarrollo:
a. Chongón-El Daular
b. El Sauce-Sabanilla-Daule
c. Samborondón - Salitre - La Aurora
d. Durán - Virgen de Fátima
e. Puerto Inca - Balao - Naranjal
f. Posorja - Golfo de Guayaquil
g. Zona Nor Oeste - Trasvase

ZONA CHONGÓN - EL DAULAR

Este eje de desarrollo está impulsado por procesos localizados: En primer lugar, la construcción del nuevo Aeropuerto Internacional de Guayaquil, situado en el borde oeste del límite urbano actual, proyecto que no sólo ampliará la capacidad de tráfico aéreo desde y hasta la ciudad, sino también, y esto de modo paralelo, promoverá la redefinición de los usos de suelo de la zona, impactado en especial en los usos de tipo habitacional.

La puesta en marcha de este proyecto está prevista para el primer quinquenio de la siguiente década (alrededor de 2025), pero su preliminar trazado ha suscitado un movimiento vertiginoso del uso del suelo en su área de influencia y, por ende, dinamizado su ocupación y transformación de la estructura de propiedad. De este modo, nuevos procesos territoriales y de práctica urbana se encuentra en pleno crecimiento. Su extensión es de aproximadamente 26.411 hectáreas (Autoridad Aeroportuaria de Guayaquil, 2009).

ZONA EL SAUCE - SABANILLA - DAULE

Este segmento se origina en la expansión urbana de la ciudad producida por los nuevos asentamientos informales que empiezan a formarse en la década de 1980 y que, en el presente, experimentan procesos de consolidación significativos.

No obstante, la característica actual del suelo urbano es su baja densidad poblacional comparada con otros sectores. Se observan aquí, por otro lado, un uso compartido industrial - habitacional e infra estructura estratégica, como la planta de captación de agua International Water Services (INTERAGUA) y la Empresa Cantonal de Agua Potable y Alcantarillado de Guayaquil (ECAPAG) denominada La Toma.

ZONA SAMBORONDÓN - SALITRE - LA AURORA

La urbanización del sector "La Puntilla" del cantón Samborondón inicia en la década de 1970 , con las migraciones de sectores socioeconómicos altos que, por primera vez desde la fundación de la ciudad, buscaban un lugar de habitación fuera de ella. El resultado se observa en el presente, como zonas habitacionales medias y altas cuyos integrantes pasan la mayor parte del día en Guayaquil, pero que ya no residen legalmente en la ciudad.

En lo básico, los cantones Durán y Samborondón son beneficiarios de población y economía por lo que, a fin de cuentas, deviene en un proceso que ha ralentizado la dinámica demográfica de la zona urbana de Guayaquil pero que, no obstante, se encuentra en su zona de influencia. En términos prácticos, se trata de uso habitacional del suelo mediante una oferta constante de vivienda para clase media. 
ZONA DURÁN - VIRGEN DE FÁTIMA

Dentro del área conurbana de Guayaquil, el único cantón con dinámica demográfica propia es Durán, cuya población actual ronda los 290.000 habitantes. La proyección oficial sitúa un crecimiento para el año 2020 que le haría alcanzar los 315.724 habitantes. Durán, al igual que Guayaquil, creció gracias a la migración. Su área de expansión actual se encuentra fuera de su zona urbana, sumamente saturada, siguiendo el curso de las autopistas troncales E40 y E49.

ZONA PUERTO INCA - BALAO - NARANJAL El proceso de expansión de la zona sur, en términos tanto demográficos como de ocupación del suelo, ha venido y viene de la mano de la agroindustria. En efecto, este sector contiene algunas de las más grandes empresas agrícolas y camaroneras del Ecuador que constantemente movilizan fuerza de trabajo $y$, por tanto, movimiento residencial. La vía troncal E26 determina la conformación o patrón de asentamiento de los principales poblados de la zona. Un fenómeno nuevo observado en estas localidades es la captación de mano de obra proveniente de la zona norte del Perú que se emplea en la minería, camaroneras y producción agrícola. Es plausible sostener que dicho proceso no está aún dimensionado (no se tienen cifras precisas al respecto).

ZONA POSORJA - GOLFO DE GUAYAQUIL

Esta zona es, de todas las analizadas, la de mayor peso o potencial de desarrollo en dimensiones diversas. En términos sociales y ambientales, se espera que, en el lapso de una década, el entorno general cambie radicalmente. El proceso de expansión de la zona suroeste, hacia Posorja, viene producido por fuertes presiones externas que suscitan nuevos cordones de desarrollo portuario, industrial y habitacional.

Por otro lado, la actual emergencia portuaria está agravada por la estandarización de naves post-panamax que demanda de un calado (profundidad de navegación) de 17,20 metros que no puede ser atendido desde el actual Puerto de Guayaquil (Fung-Sang, 2006). Articulado a estos procesos, se tiene que con la interconexión por una autopista con el aeropuerto internacional en la zona de Chongón Daular se implementaría el modelo de ínter modalismo (Fung-Sang, 2006).

El nuevo eje es también dinámico en cuanto a poblamiento y demanda inmobiliaria. Se estima que tanto el proceso de desarrollo portua- rio, aeroportuario e industrial incrementarán aún más la demanda de suelo urbano, así como su densificación produciendo, de este modo, un corredor entre la presente zona urbana de Guayaquil y el área parroquial de Posorja ( $\mathrm{Au}^{-}$ toridad Aeroportuaria de Guayaquil, 2009). Se prevé que en la siguiente década la expansión del área urbana incluya a Posorja y la densificación de la zona Samborondón - Salitre - Jújan se ralentice.

En la actualidad, a través de la vía Posorja - Progreso (E489) y la Autopista Guayaquil - Salinas (Vía Arterial E40), hay un tramo de 79,86 km hasta el sitio de El Daular. Se prevé la construcción de una variante a la E489 que evite las zonas pobladas y que se empate, recorriendo menor distancia, a la arterial E40 (Ministerio de Tranporte y Obras Públicas, 2015).

\section{ZONA NOR OESTE - TRASVASE}

Desde el año 2013, el Estado ensaya nuevas modalidades de tratamiento de los problemas generados por las ocupaciones ilegales de tierras ("invasiones", como comunmente se denominan). En términos generales estas consisten en:

- Regularización de la tenencia de PREDios ocupados ILEGALMENTE, más dotación de servicios básicos e infraestructura en las áreas de intervención. El principal caso de estudio y actuación institucional, en esta modalidad, es el sector Las Marías, en el área de Monte Sinaí;

- Reasentamiento involuntario, que se ha aplicado más o menos en situaciones en donde los asentamientos se localizan en zonas de riesgo. El caso saliente en esta modalidad es el Conjunto Residencial Ciudad Victoria.

En ambos casos, dichas intervenciones o modelos vienen patrocinados por leyes Ad Hoc aplicables casi de modo exclusivo a los sectores en donde esos procesos se implementan, lo que deja claro que los niveles de planificación no son del todo rigurosos. Un resumen de la legislación aplicada se expone a continuación:

- El Decreto Ejecutivo Nro. 433 de 21 de junio de 2007, publicado en el Suplemento del Registro Oficial Nro. 114, de 27 de junio de 2007, aprobó la Delimitación de los Espacios Geográficos Reservados, que se guardan bajo el control de las 
Fuerzas Armadas. Entre estos espacios se encuentra el Área de Reserva que protege el Canal de Trasvase Daule - Santa Elena (MIDUVI, 2014).

- El 28 de diciembre de 2010, mediante el Decreto Ejecutivo ํำ 607, se declaró como Área de Reserva de Seguridad del Sistema Hídrico de Represas y Embalses a una zona que limita con en el Distrito 8 de Guayaquil, al noroeste de la ciudad, sector conocido como Monte Sinaí, con el fin de establecer un sistema coordinado tendiente a "prevenir, minimizar y controlar riesgos, gestionar y ejecutar medidas para evitar el agravamiento de la situación geográfica en el sector" (Registro Oficial del Ecuador, 2010). Este decreto estableció una frontera para controlar la expansión de la ciudad de Guayaquil.

- El 30 de octubre de 2013 la Asamblea Nacional expidió una Ley Reformatoria a la Ley de Legalización de la Tenencia de Tierras a favor de los moradores y posicionados de predios que se encuentran en la circunscripción de los cantones Guayaquil, Samborondón y El Triunfo, conocida como Ley 88. Esta ley establece que se debe legalizar la tenencia de lotes de los moradores que se asentaron antes del 28 de diciembre de 2010, es decir, la fecha de expedición del Decreto Ejecutivo $n^{\circ} 607$. Esta ley delimitó un polígono de 743 hectáreas en Monte Sinaí, en el extremo oeste del Distrito 8 de Guayaquil. El límite norte del polígono de la Ley 88 coincide con el límite sur del polígono del Decreto Ejecutivo nº 607 (MIDUVI, 2014).

- Desde mayo de 2012 el Ministerio de Desarrollo Urbano y Vivienda (MIDUVI) inició la ejecución del "Programa Nacional de Desarrollo Urbano, Fase 1, EC-L1099”, con un contrato de préstamo suscrito entre el Banco Interamericano de Desarrollo (BID) y el Ministerio de Finanzas bajo el Nro. 2679/OC-EC. Uno de los principales componentes del programa fue el reasentamiento involuntario de 397 familias que construyeron sus viviendas dentro del Área de Reserva de Seguridad antes de la fecha de expedición del Decreto Ejecutivo n ${ }^{\circ}$ 607 (diciembre 2010). Asimismo, se identificaron 1252 familias de invasio- nes en el Área de Reserva de Seguridad a los que se determinó como potenciales demandantes de vivienda con subsidios del MIDUVI. En términos generales, en el polígono de la Ley 88 se identificaron 16.000 construcciones, de las cuales 12.000 eran viviendas con una frecuencia de 4 personas por hogar, lo cual daba a entender que la población total era de aproximadamente 50.000 personas. La actualización posterior identificó una población objetivo de 3622 familias asentadas en 440 hectáreas del Área Reservada de Seguridad (ARS) (MIDUVI, 2014).

Con los antecedentes citados, es posible determinar que los hogares que fueron incluidos en ciudad Victoria estuvieron localizados en:

a. ARS;

b. Área de la franja de protección del canal de trasvase de Santa Elena en el sector de Monte Sinaí, que comprende un área de 309 hectáreas;

c. Zona de riesgos en el Noroeste de la ciudad de Guayaquil, declaradas en estado de emergencia o con informe técnico de la Secretaria Nacional de Gestión de Riesgos.

d. Las viviendas que componen ciudad Victoria son departamentos con una superficie de $40 \mathrm{~m}^{2}$, contenidos en bloques de dos pisos, con dos habitaciones. Cuenta con una cierta infraestructura y servicios básicos, tales como: agua potable, alcantarillado instalaciones eléctricas y telefónicas (MIDUVI, 2014).

Los hogares desplazados en Las Marías a los que MIDUVI les asignó un solar para que puedan volver a edificar su vivienda se vieron en la necesidad de asumir todos los gastos del proceso, tales como desarmado de su antigua vivienda para reutilización de materiales, traslado y construcción de su nueva casa. Estas actividades fueron financiadas, por un lado, con horas de trabajo de los adultos y jóvenes; y con parte de los ingresos del hogar, en segundo lugar.

En términos generales, estas experiencias de tratamiento de la periferia pobre, no observadas en ningún proceso anterior, tienen ya resultados ciertamente interesantes y contradictorios a la vez (Figura 2). 


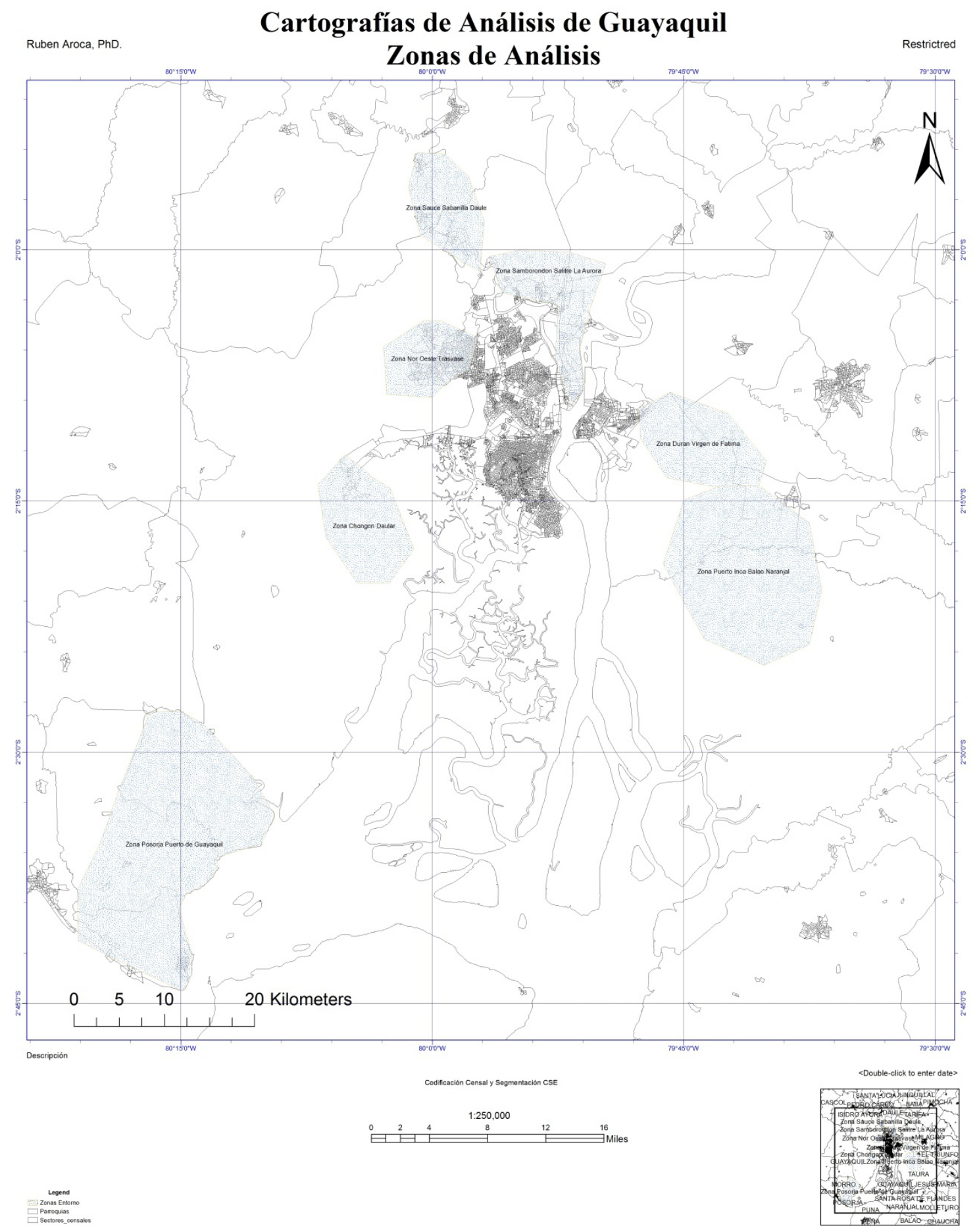

Figura 2. Zonas de desarrollo analizadas.

ALGUNAS LÍNEAS DE CONCLUSIÓN

Los procesos de desarrollo urbano analizados (en términos de relaciones socioeconómicas, de uso de suelo y simbólicas) tienen lugar en Guayaquil, su área urbana y los cantones dentro del área conurbana. El patrón histórico de asentamiento y crecimiento de Guayaquil ha sido, y en cierto modo aún es, de tipo ribereño. Esto significa que antes que cualquier otro tipo de suelo, los que se vieron inicialmente ocupados y saturados fueron justamente aquellos que permitían el contacto directo de la población con el río. Esta relación que ha sido vital para la ciudad, al mismo tiempo supuso un tipo de acción antrópica irreversible, que actualmente influye de modo directo y negativo en la gestión de los riesgos relacionados especialmente con la época de lluvias.

La elevada demanda de suelo urbano en Guayaquil, así como el aumento de la densidad 
poblacional del área consolidada, produjo a comienzos de la década de 1970 que los habitantes optaran por ofertas habitacionales fuera del área urbana-cantonal. Estas posibilidades estuvieron al alcance, en principio, solo para estratos de alto nivel de consumo, pero ya en los años 90 la clase media encontró alternativas más viables en esas mismas zonas. De este modo, fue posible la dinamización demográfica de los cantones Samborondón, Durán, e incluso Daule que, no obstante, supuso para Guayaquil el surgimiento de una "población flotante" de no-residentes que diariamente llegan a la ciudad, presionan sobre los servicios y por la noche regresan a sus conjuntos habitacionales fuera del área urbana o cantonal.

La migración intra e interurbana de la clase media y los sectores de alto consumo no impidió la saturación de actividades en las zonas del centro urbano o adyacente a las zonas ribereñas. Este fenómeno está fuertemente relacionado al surgimiento de nuevas áreas habitacionales en los cantones del entorno inmediato a las zonas urbanas más dinámicas de la ciudad, pues con bastante frecuencia los nuevos conjuntos residenciales se ubicaron en áreas aledañas a las márgenes de los ríos Daule y Babahoyo.

La zona Guayaquil - Posorja es, de todas las analizadas, la de mayor peso o potencial de desarrollo en dimensiones diversas. En términos sociales y ambientales se espera que, en el lapso de una década, el entorno general cambie radicalmente. El proceso de expansión de la zona suroeste hacia Posorja, viene producido por fuertes presiones externas que suscitan nuevos cordones de desarrollo portuario, industrial y habitacional.

Este eje es también dinámico en cuanto a poblamiento y demanda inmobiliaria. Se prevé que en la siguiente década la expansión de área urbana incluya a Posorja y la densificación de la zona Samborondón - Salitre-Jújan se ralentice.
Finalmente, es posible sostener que los nuevos sectores pobres que inicialmente se formaron a la manera de las "invasiones" o tomas ilegales de tierra en la periferia urbana (nor - oeste), en los últimos años han observado un proceso, al menos parcial, de reordenamiento en forma de reasentamientos involuntarios o regularización de la posesión.

Dichos reasentamientos o desplazamientos se han producido en la intersección entre los asentamientos irregulares con las obras de infraestructura urbana de tipo público, por un lado, o como una acción necesaria del Estado cuando se trata de viviendas construidas en las áreas reservadas para seguridad de los canales y el trasvase.

\section{REFERENCIAS BIBLIOGRÁFICAS}

Autoridad Aeroportuaria de Guayaquil (2009). Plan de Desarrollo Integral de la Zona del Aeropuerto Daular-Chongón. Guayaquil: AAC.

Cieza de León, P. D. (2005). Crónica del Perú. El Señorío delos Incas. Caracas: Biblioteca Ayacucho.

Espinoza Soriano, W. (1999). Etnohistoria Ecuatoriana: Estudios y Documentos. Quito: Abya - Yala.

Fung-Sang, M. (2006). Factibilidad y urgente necesidad del Puerto de Aguas Profundas para Guayaquil. Revista Tecnológica ESPOL, Vol. 20.

Hamerly, M. (2006). Recuentos de dos ciudades: Guayaquil en 1899 y Quito en 1906. Procesos. Revista Ecuatoriana de Historia, 135 - 163.

INPC. (2017). Sistema de Información del Patrimonio Cultural Ecuatoriano (SIPCE). Guayaquil, Guayas, Ecuador: INPC.

MIDUVI. (2014). Programa de Desarrollo Urbano. Guayaquil: MIDUVI.

Ministerio de Tranporte y Obras Públicas. (2015). Cartografía Vial. Quito: MTOP.

Registro Oficial del Ecuador. (28 de Diciembre de 2010). Decreto Ejecutivo 607. Decretos. Quito, Pichincha, Ecuador: RO. 